\title{
Involuntary attentional capture by abrupt onsets
}

\author{
ROGER W. REMINGTON and JAMES C. JOHNSTON \\ NASA-Ames Research Center, Moffett Field, California \\ and \\ STEVEN YANTIS \\ Johns Hopkins University, Baltimore, Maryland
}

\begin{abstract}
The extent to which brief abrupt-onset visual stimuli involuntarily capture spatial attention was examined in five experiments. The paradigm used was intended to maximize the opportunity and incentive for subjects to ignore abrupt-onset distractor stimuli in nontarget locations. Subjects made a speeded two-choice response to a target letter appearing in one of four boxes. An abrupt-onset visual stimulus, easily discriminable from the target, was flashed briefly prior to the presentation of a target. In separate blocks, the flash stimulus marked the box in which the target would subsequently appear (SAME), a different box (DIFF), fixation (CENTER), or all four boxes (ALL). Prior to each block, subjects were informed of the flash-target relationship. In all five experiments, response time was elevated in the DIFF, CENTER, and ALL conditions. The interference effect was larger for the DIFF condition and persisted for longer flash-target SOAs. These results suggest that, under appropriate conditions, spatial attention can be involuntarily drawn to abrupt-onset events despite the intention of subjects' to ignore them.
\end{abstract}

Relevant visual information occurs in a variety of unpredictable locations, making it necessary to search or sample from a number of spatially separate sources. Research over the last two decades has demonstrated that visual search is accomplished not only by saccadic eye movements, but also by the covert allocation of spatial attention. Saccadic eye movements are, in fact, often preceded by shifts of spatial attention to the saccade target, and there is evidence that the control of spatial attention involves separate neural mechanisms (e.g., Bushnell, Robinson, \& Goldberg, 1978; Posner, 1980; Remington, 1980).

The control of spatial attention is complex, in part because it must be responsive to both endogenous and exogeneous factors (Posner, 1980). Endogenous factors are associated with voluntary, cognitively driven shifts of spatial attention aimed at achieving an immediate visual goal, such as finding the book with a certain title. Exogeneous factors are associated with involuntary, stimulus-driven shifts of spatial attention that alert one to the occurrence of an external event, such as a sudden flash of light or movement in the periphery (e.g., Müller \& Rabbitt, 1989; Posner, 1980; Yantis \& Jonides, 1984). The allocation of spatial attention appears to be jointly determined by these potentially conflicting demands. What happens when endogenous and exogeneous demands directly conflict? Can

\footnotetext{
We would like to thank Charles Folk for useful discussions concerning this work, as well as C. W. Eriksen, Harold Pashler, and two anonymous reviewers who provided helpful comments on earlier drafts of this paper. S. Yantis participated with the support of NASA JRI NCA2-413 and NIMH Grant ROI-MH43924. Correspondence may be addressed to R. Remington, NASA-Ames Research Center, MS 262-2, Moffett Field, CA 94035 (e-mail: roger@eos.arc.nasa.gov).
}

voluntary attentional control, in accord with a subject's intentions, prevent involuntary attentional responses to sudden changes in visual stimulation? In this paper, we investigate these questions by examining observers' ability to ignore potentially distracting visual stimulation.

Empirical support for voluntary control of spatial attention by endogenous factors comes from demonstrations that spatial attention can be allocated in response to a person's immediate goals. Voluntary control has been observed in experiments that use a centrally presented symbolic cue to indicate which of several stimulus locations is most likely to contain a target. When the target occurs at the cued location, there are performance enhancements (or benefits) for both reaction time (e.g., Eriksen \& Hoffman, 1972; Posner, 1980; Posner, Snyder, \& Davidson, 1980; Remington \& Pierce, 1984; Shulman, Remington, \& McLean, 1979) and accuracy (e.g., Bashinski \& Bacharach, 1980; Eriksen \& Yeh, 1985; Hawkins et al., 1990; Hoffman \& Nelson, 1981; Müller \& Findlay, 1987). These enhancements are generally accompanied by performance impairments (or costs) when the target occurs at an uncued location (but see Jonides \& Mack, 1984). This pattern of cost and benefit is consistent with the hypothesis that attention has been directed in response to the central cue. It is important to note that the mere presence of the central cue, without subjects' desire to use it, does not produce costs and benefits (i.e., if the central cue is uncorrelated with target location, as in Jonides, 1981). Thus, with central cues, it appears that attention is shifted voluntarily to the indicated location in response to the observer's intent. ${ }^{1}$

In contrast, for shifts of attention to be involuntary they would have to occur without subjects' deliberate intent; 
to be strongly involuntary, attentional shifts would have to occur despite attempts to prevent them. Empirical evidence linking exogeneous factors with involuntary shifts of spatial attention has come from demonstrations that attention is drawn to the location of abruptly onset visual stimuli even when such responses are suboptimal. ${ }^{2}$ When an abrupt-onset stimulus occurs at one screen location followed, after a brief interval, by a target at another location, target detection (e.g., Posner \& Cohen, 1984) and discrimination (e.g., Jonides, 1981; Lambert, Spencer, \& Mohindra, 1987; Müller \& Rabbitt, 1989; Warner, Juola, \& Koshino, 1990) are impaired even though the location of the abrupt-onset stimulus is not a valid predictor of target location. These results are consistent with the hypothesis that abrupt-onset stimuli disrupt ongoing processing by involuntarily capturing attention.

To conclude that a stimulus has involuntarily captured attention, it is necessary to show that it has influenced processing when the subject intended for it not to do so. This requires a situation in which it is clearly and unequivocally in the subject's interest to prevent a certain class of stimuli from controlling attention. One cannot conclude from previous studies that abrupt onsets capture attention in a strongly involuntary fashion, because no previous study has met this condition. Attentional capture by abrupt-onsets has been observed under conditions in which the abrupt onset (1) is itself the target on some of the trials (Yantis \& Jonides, 1984), (2) correctly corresponds with target location on some proportion of the trials (Müller \& Rabbitt, 1989; Posner \& Cohen, 1984), or (3) is otherwise task relevant (Warner et al., 1990). Attentional capture cannot be conclusively demonstrated to be involuntary by showing that abrupt onsets that only occasionally correspond to the target location (e.g., $20 \%$ valid) nonetheless interfere with performance. Voluntary strategies, such as probability matching (e.g., Eriksen \& Yeh, 1985), can also produce such results, because subjects may use (and therefore attend to) the abrupt onset on a similar proportion of trials. Thus, the results of studies using low-validity abrupt-onset precues do not address the strong claim that it is not possible to voluntarily inhibit an attentional response.

Yantis and Jonides (1990) have identified one set of conditions in which an abrupt onset at a nontarget location does not seem to draw spatial attention away from the target location. They had subjects search for a prespecified target letter in an array of four letters equidistant from fixation. Three of the letters were revealed by removing segments from composite figures that were visible throughout the trial (referred to as no-onset letters). The fourth letter was abruptly onset at a previously empty location. In one experiment (Yantis \& Jonides, 1990, Experiment 3), a central cue was presented $200 \mathrm{msec}$ prior to the presentation of the target set. The validity of the cue was varied in separate blocks (validities of $25 \%, 75 \%$, and $100 \%$ were used) to manipulate the degree to which attention was strongly focused before the letters appeared. If attention were drawn involuntarily to the onset, there should be a response time (RT) advantage for abrupt-onset targets over no-onset targets, regardless of the attentional set of the subject. The results revealed an advantage for onset targets in all conditions except in the $100 \%$ valid condition, where performance was identical for onset and no-onset targets. Thus, subjects were able to prevent attention from being drawn to the abrupt-onset distractor only when (1) they knew with certainty the location in which the target would occur and (2) they were given sufficient time to focus their attention on that location in advance. These results indicate that involuntary attentional capture by abrupt onsets is not inevitable under all circumstances: when attention is successfully focused in one location, capture by an abrupt onset can be prevented. However, this finding does not reveal whether it is possible, given sufficient inducement, to voluntarily withhold an attentional response in the absence of a focused attentional set elsewhere.

Our criterion for the involuntariness of attentional capture rests on the inability of the internal processes controlling attention to resist a distracting stimulus. This inability is one of the criteria for classifying a process as strongly automatic (e.g., Jonides, 1981; Jonides, NavehBenjamin, \& Palmer, 1985; Kahneman \& Treisman, 1984; LaBerge, 1981; Shiffrin \& Schneider, 1977). Thus, the determination of whether or not attentional shifts occur involuntarily provides an important clue to the larger theoretical issue of whether or not attentional shifts occur automatically. Whether or not attentional capture satisfies further criteria for automaticity in addition to involuntariness is, however, beyond the scope of this paper (see Yantis \& Jonides, 1990).

The goal of the present experiments, then, was to rigorously test the extent to which abruptly onset visual events capture attention involuntarily. The key to this goal is maximizing the opportunity and incentive for subjects to ignore abrupt-onset stimuli without having them first focus attention on any one potential target location. In our experiments, the presentation of a target letter in one of four screen locations was preceded by a brief abrupt-onset stimulus, which we will refer to as the flash stimulus. In the distraction conditions, the flash always occurred at a location other than where the target would occur. To maximize the opportunity and incentive to ignore the distracting flash, the subjects were informed of the spatial relationship between the flash and the target and were told not to let it impair their performance. The relative location of the target and the flash stimulus was varied across blocks, but within a block the spatial relationship between target and flash remained consistent (in fact, consistently misleading in the distraction condition).

The primary indicator of attentional capture is the observed increase in discrimination latency-or interference-in the distraction conditions relative to that in the baseline conditions. If it is possible to voluntarily inhibit an attentional response to an irrelevant abrupt onset, then the flash stimuli in the distraction conditions should produce no interference. Persistent interference from flash 
stimuli in the distraction conditions would provide strong support for the involuntary capture of spatial attention by abrupt onsets.

\section{EXPERIMENTS 1-3}

Experiment 1 included several design features intended to maximize the opportunity and incentive to ignore the flash stimulus in the distraction conditions. First, the relationship between the location of the flash stimuli and subsequent target locations was held constant within a block. In the distraction conditions, targets never occurred at the location of the preceding flash stimulus. Second, the subjects were informed of the spatial relationship between flash stimuli and targets before each block. Third, flash and target stimuli were constructed to minimize the likelihood that focal attention would be required to discriminate between them. Targets and flash stimuli could be further discriminated by order of appearance, since the order of events within a trial never varied within a block. Finally, because the task required target discrimination, the abrupt onset common to both flash stimuli and targets did not provide a sufficient basis for responding.

In Experiments 2 and 3, we incorporated changes to the design of Experiment 1, enabling us to explore the magnitude and generality of the results. In Experiment 2, a random foreperiod was added to the fixed foreperiod of Experiment 1 so that flash onset time could not be predicted with certainty. In Experiment 3, new flash and target stimuli were used to ensure that the results were not due to the specific physical properties of the stimuli used in Experiment 1. Table 1 summarizes the important differences between the first three experiments.

\section{Method}

\section{Experiment 1}

Subjects. The subjects were either drawn from the NASA-Ames student and postdoctoral support staff or recruited from the local population. Eighteen subjects participated in Experiment 1. They ranged in age from 18 to 41 years, and each had normal or correctedto-normal vision. All were paid for their participation.

Apparatus and Stimuli. Testing took place in a darkened, soundattenuated chamber. Trial sequence, timing, and data collection were controlled by a $12-\mathrm{MHz} 68000$-based microcomputer. Stimuli were displayed on a 21-in. diagonal, calligraphic CRT display with a P4 phosphor at a viewing distance of $69 \mathrm{~cm}$. The CRT was driven by a Hewlett-Packard Model $\mathbf{4 0 0 0}$ vector generator. Photometer measurements were used to verify all presentation times. Figure 1 shows the size and arrangement of display items. Targets and flash stimuli were constructed to differ in elementary features to max- imize their dissimilarity. The flash stimulus was reticle, consisting of four terminated straight lines, forming a cross with the center missing. The targets were the characters " 8 " and " 0 ," both closed figures composed of curved lines.

Design. Four of the five conditions that defined the spatial relationship between the targets and the flash stimuli are shown graphically in Figure 2. The CENTER and ALL conditions involved the same flash stimuli as the same-box (SAME) and different-box (DIFF) conditions, but the onsets provided no information as to the location of the ensuing target. There was an additional no-flash (NONE) condition, not shown in Figure 2, in which the target was presented without a preceding flash stimulus. Note that for the DIFF condition the location of a flash stimulus never contained a subsequent target, whereas for the SAME condition the target always occurred in the location indicated by the flash stimulus.

The five conditions were varied across blocks within subjects. The subjects were tested in two 40 -min sessions separated by a 10-min break. Each session consisted of the same order of six experimental blocks (SAME, DIFF) and three control blocks (NONE, CENTER, ALL). The subjects were randomly assigned to one of six presentation orders. Control conditions always appeared in Blocks 1, 4, and 7; experimental conditions in the intervening blocks alternated in order such that no two adjacent blocks had the same experimental condition. Across the six orders, each control condition occurred twice in each of the three possible positions, with the order of presentation of the experimental condition reversed. Thus, the DIFF and SAME conditions occurred equally often in positions $2,3,5,6,8$, and 9 , and preceded and followed each control condition equally often. The subjects completed two runs through the same order condition for a total of 18 blocks per testing session.

Each block consisted of 48 experimental trials, with two warmup trials at the beginning of the block to allow the subjects to adjust to the new condition. The warm-up trials were excluded from analysis. Error trials were presented again at the end of a block. A block ended when the subjects correctly responded to all of the 48 original trials.

Procedure. Written and oral descriptions of the displays and procedures were used to familiarize the subjects with the task. The blocked arrangement of the flash-stimulus conditions was emphasized. The instructions stressed that the primary goal was to respond as quickly and accurately as possible to the target letters. The subjects were told to ignore the flashes if they interfered with target RT. They were instructed to use the RT feedback at the end of each trial as a performance metric and to minimize that RT. The instructions stressed that eye fixation should be maintained; it was explained that events occurred so rapidly that eye movements would interfere with performance. When it was clear that the instructions had been understood, the subject was taken to the testing booth and, with the experimenter present, was tested on one practice block of NONE trials. The experimenter then gave final instructions, left the booth, and the experimental sequence began.

The beginning of each block was signaled by the presentation of one of the descriptors, SAME BOX, DIFFERENT BOX, NONE, ALL, or CENTER, indicating the appropriate condition for that block. A keypress extinguished the descriptor and began the trial

Table 1

Independent Variables Used in Experiments 1, 2, and 3

\begin{tabular}{|c|c|c|c|}
\hline Experiment & Foreperiod & Flash Stimuli & Targets \\
\hline 1 & Fixed & Reticles & 8,0 \\
\hline & (400 msec) & (Terminated Straight Lines) & (Closed Curved Lines) \\
\hline 2 & Random & Reticles & $\begin{array}{c}8,0 \\
\text { (Closed Curved Lines) }\end{array}$ \\
\hline & $(\mu=700 \mathrm{msec})$ & (Terminated Straight Lines) & $\begin{array}{c}\text { (Closed Curved Lines) } \\
x=\end{array}$ \\
\hline 3 & $\begin{array}{c}\text { Random } \\
(\mu=700 \mathrm{msec})\end{array}$ & $\begin{array}{c}\text { Circles } \\
\text { (Closed Curved Lines) }\end{array}$ & $\begin{array}{l}\mathrm{x},= \\
\text { (Terminated Straight Lines) }\end{array}$ \\
\hline
\end{tabular}




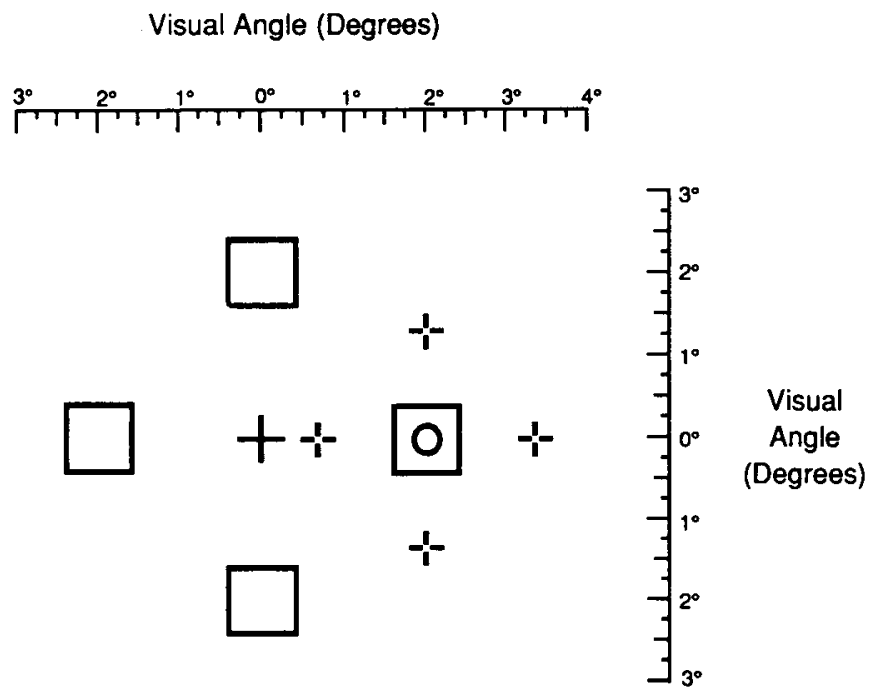

Experiments 182 Experiments 3.4.8.5

Visual Angle (Degrees)

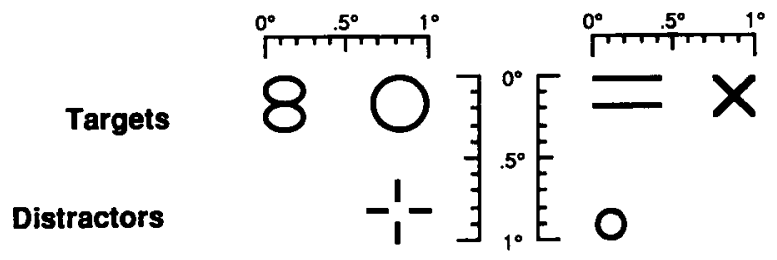

Figure 1. Display layout and stimulus dimensions for Experiments 1-5. The top panel illustrates a trial from the SAME condition in Experiment 1.

sequence. The subjects were instructed to position themselves in the chinrest prior to the start of a block and to remain so positioned for the duration of the block. At the end of a block, the word REST appeared for $15 \mathrm{sec}$. During this time, the subjects were encouraged to relax in preparation for the next block. The experimenter monitored performance from an adjacent room and used the interblock interval to communicate performance information. The subsequent block could be started only after the rest period ended and the screen displayed the description of the next block type. Thus, the subjects were required to take a $15-\mathrm{sec}$ break between blocks but could choose to rest longer.

The sequence of events on each trial is illustrated in Figure 3. A trial began with the simultaneous onset of the central fixation cross and surrounding boxes, followed $400 \mathrm{msec}$ later by a $50-\mathrm{msec}$ blanking of the fixation cross (fixation blink). There was a fixed 400-msec foreperiod between the fixation blink and the onset of the flash stimulus. The duration of the flash stimulus was $24 \mathrm{msec}$. In the NONE condition, no flash was presented and the 24-msec presentation interval was maintained with no change in the display. The time between flash onset and target onset (stimulus-onset asynchrony, or SOA) was $120 \mathrm{msec}$. The target appeared for $24 \mathrm{msec}$ in one of the four boxes. The subjects pressed one of two keys with the index finger of the left or right hand to indicate which target letter had appeared. Since eye position was not monitored, stimulus durations and SOAs were chosen to minimize the probability of an eye movement prior to target offset. Error trials were presented again at the end of the block. RTs greater than 1,500 msec were considered errors, and those trials were rerun. Feedback was provided following the subject's response. The RT (in milliseconds) was presented following correct responses; the word error was presented following incorrect responses. Feedback duration was $500 \mathrm{msec}$, with a subsequent intertrial interval of 700 msec.

\section{Experiment 2}

In Experiment 2, and in all subsequent experiments, a random foreperiod was added to the fixed 400 -msec foreperiod of Experiment 1 . There was a constant 0.3 probability that the foreperiod would end in each 100 -msec interval following the fixed 400 -msec foreperiod. This is equivalent to a foreperiod chosen randomly from a geometric distribution with a mean of $300 \mathrm{msec}$ to which a constant $\mathbf{4 0 0} \mathrm{msec}$ has been added. The mean total foreperiod duration was thus $700 \mathrm{msec}$. In all other respects, the conditions in Experiment 2 were identical to those in Experiment 1. Eighteen new subjects, selected as in Experiment 1, were tested.

\section{Experiment 3}

In Experiment 3, and in all subsequent experiments, the flash stimulus was a set of small circles, four closed figures composed of curved lines; the targets were the characters " $x$ " and " =," both composed of terminated straight lines. Thus, the contrast between 


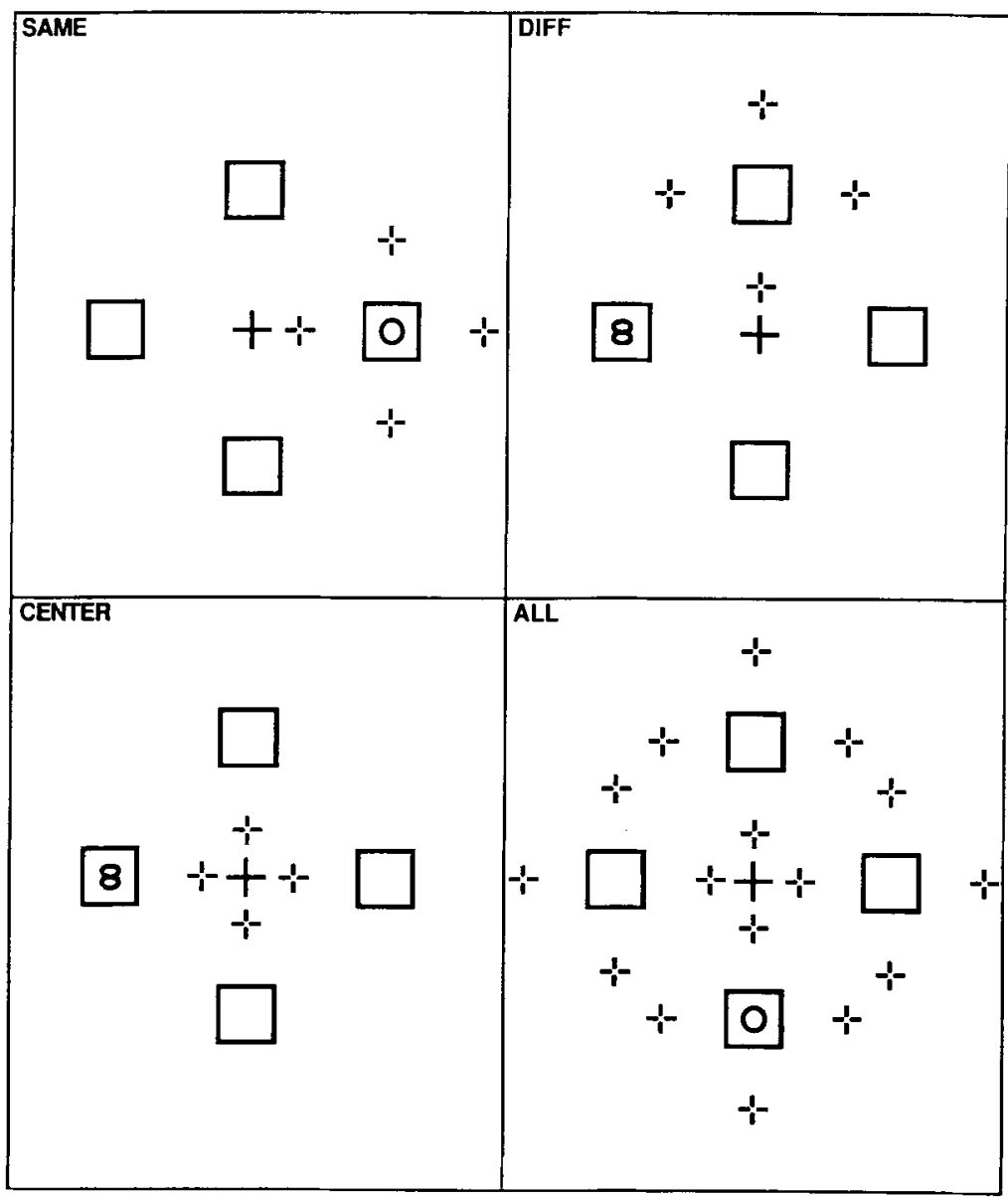

Figure 2. The arrangement of targets and pretarget flashes in the four flash conditions of Experiment 1. Note that flashes and targets never appeared simultaneously.

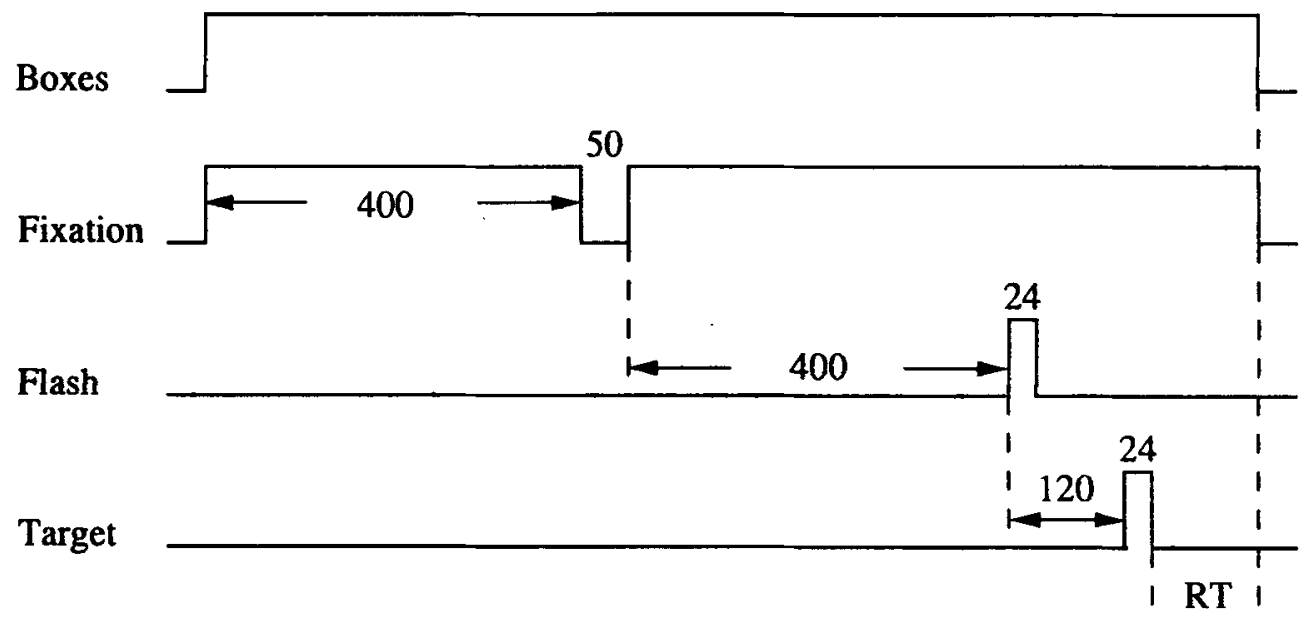

Duration (ms)

Figure 3. Sequence of events in Experiment 1. For each stimulus, a downward deflection indicates that it is off and an upward deflection means that it is on. 
Table 2

Mean Response Time (RT) and Percent Errors (PE) for Each Condition in Experiments 1, 2, and 3

\begin{tabular}{|c|c|c|c|c|c|c|c|c|c|c|}
\hline \multirow[b]{2}{*}{ Experiment } & \multicolumn{2}{|c|}{ SAME } & \multicolumn{2}{|c|}{ NONE } & \multicolumn{2}{|c|}{ ALL } & \multicolumn{2}{|c|}{ CENTER } & \multicolumn{2}{|c|}{ DIFF } \\
\hline & RT & PE & RT & PE & RT & $\mathrm{PE}$ & RT & $\mathrm{PE}$ & RT & PE \\
\hline 1 & $483^{a}$ & 5.43 & $498^{b}$ & 3.17 & $504^{b, c}$ & 5.86 & $512^{c}$ & 5.38 & $511^{c}$ & 5.05 \\
\hline 2 & $496^{a}$ & 5.6 & $497^{a}$ & 4.03 & $520^{\mathrm{b}}$ & 5.67 & $524^{b}$ & 6.23 & $531^{\mathrm{b}}$ & 4.54 \\
\hline 3 & $524^{\mathrm{a}}$ & 4.53 & $533^{a}$ & 3.63 & $553^{b}$ & 4.58 & $557^{b}$ & 4.93 & $561^{b}$ & 4.42 \\
\hline
\end{tabular}

Note-For each experiment, response times with the same superscript did not differ significantly from one another.

the elementary features of the target and the flash established in Experiments 1 and 2 was maintained in Experiment 3, but the assignment was reversed. Twelve new subjects, selected as before, were tested. In all other respects, conditions in Experiment 3 were identical to those in Experiment 2.

\section{Results}

Table 2 shows the mean RT and error percentage for each of the flash conditions for Experiments 1, 2, and 3. Analyses of variance (ANOVAs) were performed on the mean correct RTs and the arcsin transform of the error proportions for the five conditions (NONE, CENTER, ALL, DIFF, SAME) separately for each experiment. ${ }^{3}$ Post hoc analyses were Newman-Keuls tests with $\alpha=$ .05 . The results of the analyses will be reported separately for each experiment.

\section{Experiment 1}

The ANOVA on the correct RTs showed a significant main effect of condition $[F(4,68)=8.58, p<.001]$. Post hoc analysis showed that SAME RTs were significantly lower than those in all conditions; CENTER and DIFF RTs were significantly higher than those in the NONE condition. An ANOVA on the arcsin transform of the square root of the proportion of errors showed a significant effect of condition $[F(4,68)=4.56, p<$ $.005]$. Post hoc analysis showed that the error rate in the NONE condition was significantly lower than in the flashstimulus conditions. No other comparisons were significant. We cannot rule out a speed-accuracy tradeoff that might have elevated RTs in the NONE condition relative to those in the flash conditions. (Note that, if a speedaccuracy tradeoff did elevate RTs in the NONE condition, then the true magnitude of the RT costs in the ALL, CENTER, and DIFF conditions is larger than indicated by the raw $R T$ differences).

\section{Experiment 2}

The ANOVA on the mean RTs from Experiment 2 revealed a significant effect of condition $[F(4,68)=10.51$, $p<.001]$. Post hoc analysis showed that RTs in the SAME and NONE conditions were significantly faster than RTs in the ALL, CENTER, and DIFF conditions. There were no significant differences within these clusters. An ANOVA on the arcsin transform of the proportion of errors failed to show a significant effect $[F(4,68)=$ $2.47, p<.053]$. The effect almost reached significance, and, as in Experiment 1, the NONE condition had the lowest error percentage.

\section{Experiment 3}

The ANOVA on the mean correct RTs from Experiment 3 showed a significant effect of condition $[F(4,44)$ $=6.50, p<.001]$. Post hoc analysis showed the same pattern as that in Experiment 2; RTs in the SAME and NONE conditions were significantly faster than in the ALL, CENTER, and DIFF conditions. An ANOVA on the transformed error proportions failed to show any significant differences in error rate between the conditions $[F(4,44)=1.02, p<.5]$

\section{Discussion}

In all three experiments, performance disruptions were found when a target was preceded by an abrupt onset (flash stimulus) at another location. The location of the flash relative to the target was blocked so that the subjects knew the onsets would always (SAME condition) or never (DIFF condition) occur at the location of the upcoming target. Despite the clear incentive to ignore the distracting effects of these stimuli in the DIFF condition, discrimination was significantly slowed. This provides strong evidence that voluntary inhibition of attentional capture by abrupt onsets is not possible under the conditions tested here. There was an almost equally large disruption for the CENTER condition in which, for a complete block, the abrupt-onset stimulus occurred around the fixation point. The critical feature common to the two conditions was the presence of a spatially specific pretarget onset stimulus at a position that the subjects knew would not contain a target. The disruption for the CENTER condition suggests that it is not possible to ignore an abrupt-onset stimulus even given knowledge of exactly where it will occur and even when that location never contains a target. It is possible, of course, that the requirement to maintain fixation at the same location as the onset in the CENTER condition makes it difficult to implement any strategy that would exclude an attentional response to events at fixation.

One might argue that the reason the flash could not be ignored in the critical DIFF condition was because it could not easily be discriminated from the target stimulus to which a response was required. This is a valid criticism of simple reaction time studies (e.g., Posner \& Cohen, 1984), where target onset, not identity, is the property to which subjects must respond and subject responses are 
based on a property that is shared between target and distractor. There are several reasons for rejecting this account of our results. First, our task required a discrimination response; target onset was not the relevant property. Second, the present results were obtained under conditions in which the discrimination between the target and the flash could readily be made preattentively (see Treisman \& Gormican, 1988); the flash stimuli and the targets differed substantially in their visual feature composition (see Figure 1): the discrimination of flash from target required a discrimination between closed, curved figures and terminated, straight line figures. Third, the experimental procedures guaranteed both spatial and temporal separation of flashes and targets; the flash stimuli always occurred outside a box, the targets within, and the order of events was fixed, with the distractor always occurring first. Finally, in the CENTER condition, the flash stimuli always occurred at a known fixed location around fixation. Together, these facts undermine the argument that the subjects were unable to avoid attending to the abrupt-onset stimuli because they could not easily be discriminated from targets.

The ALL conditions of Experiments 2 and 3 produced interference approximately equal to that in the CENTER condition. It is tempting to conclude that the diffuse, spatially distributed cuing pattern in the ALL condition resulted in a distribution of attention to a correspondingly wide area of the visual field, and that this allocation of attention produced the interference. However, the difference in luminance between the 16 flash stimuli in the ALL condition and the 4 flash stimuli in the CENTER and DIFF conditions makes it impossible to rule out the contribution of other factors (such as masking) in producing the interference.

The differences between experiments provide useful information on the robustness of the interference effect and the potential for control by subject strategies. The results appear robust to changes in the physical properties of the abrupt-onset stimulus. In Experiment 3, for example, the elementary feature distinction between flash stimuli and targets was maintained (straight line, terminated vs. curved, closed), but reversed (see Figure 1). In Experiments 1 and 2 , the flash stimulus contained terminated, straight line segments. The similarity in the results of Experiments 2 and 3 show that the results do not depend on the relative shapes of the target and flash stimuli.

The results also suggest that attentional capture is robust to changes in temporal uncertainty. The intent behind Experiment 1 was to provide a very regular sequence of events in order to maximize the opportunity for voluntary control of spatial attention. Thus, a fixed 400 -msec foreperiod was used. However, the temporal regularity created the possibility that time alone could provide a basis for a temporal filtering of sensory input, a strategy that would rarely be possible outside of rigid laboratory experimental paradigms. Experiment 2 tested the possibility that involun- tary attentional capture would be even stronger when such temporal filtering was made more difficult.

A comparison of response times in Experiments 1 and 2 suggests that the primary effect of the random foreperiod was to increase RT for all conditions containing flash stimuli without changing performance in the NONE condition. This is consistent with the hypothesis that the fixed interval between the flash stimuli and targets in Experiment 1 produced a temporal predictability that enabled the use of time-based filtering to reduce interference. Because RT in the SAME condition is also elevated in Experiment 2, though not as much as the DIFF condition, increased temporal uncertainty apparently made it both more difficult to use the flash stimuli voluntarily and more difficult to ignore them. It is not clear whether temporal filtering might alter the early, preattentive processing of the visual event or affect later stages concerned with the control of spatial attention. We cannot reject the hypothesis that some limited control over spatial attention is possible when confronted with abrupt onsets, and that increased temporal certainty enhances this control. However, though the magnitude of the effect is influenced by temporal uncertainty, the results of Experiment 1 are clear evidence that interference from irrelevant abrupt onsets does not require temporal uncertainty.

Error rates for the NONE condition were consistently lower than for the flash conditions, although this is only statistically significant in Experiment 1. This could have been the result of a speed-accuracy tradeoff produced by the altering properties of the abrupt-onset flashes. There is evidence that precues increase altertness, which, in turn, leads to faster responding and higher error rates (see Posner, 1978, chapter 5). It is important to note that the effect of such a tradeoff in these experiments would have been to reduce the measured magnitude of the interference effect by elevating RT in the NONE condition. Thus, any speed-accuracy tradeoffs would result in an underestimate of the interference effects critical to the conclusions of this article.

The main purpose of Experiments 1-3 was to study cost in the $100 \%$ invalid DIFF condition; comparisons between other conditions are of secondary interest, since their main function was to provide difference baseline conditions for assessing cost in the DIFF condition. However, it is worth noting that only in Experiment 1 was there a significant benefit for the $100 \%$ valid (SAME) condition when compared with the NONE condition. Even in Experiment 1, the evidence of benefit for the valid flashes is accompanied by a lower error rate in the NONE condition than in the SAME condition, raising the possibility of a speedaccuracy tradeoff. One might have expected that the spatial and temporal information provided the abrupt onset in SAME blocks would have led to measurable benefits over a no-cue baseline. It should be noted that in spatial cuing studies such as ours, the critical comparison is usually between valid (SAME) and invalid (DIFF), and 
not with a no-cue (NONE) condition (see Jonides \& Mack, 1984 , for a discussion of the difficulties in selecting appropriate baseline conditions). Therefore, our results may not be atypical, since we find a consistent advantage for SAME over DIFF RTs.

The relative performance of the SAME and NONE conditions could indicate the presence of some generalized interference produced by the cues (flashes) that is affecting performance in all cued conditions. Related effects have been described by Eriksen and Schultz (1978) as cognitive masking and by Kahneman, Treisman, and Burkell (1983) as filtering cost. Both the cognitive masking and the filtering cost hypotheses emphasize an increase in processing time due to the presence of additional stimuli (the flash in our experiments; see Eriksen \& Schultz, 1978). Such an effect, however, would be expected to act uniformly across the visual field and would not produce the advantage for the SAME condition over the DIFF condition, which was consistently obtained in Experiments 1-3. For example, the introduction of a random foreperiod in Experiment 2 had the effect of elevating RT for all cued conditions, but it did not alter the significant benefit for SAME responses relative to that for DIFF responses. Rather, the consistent difference between the DIFF and SAME conditions provides strong evidence for a positionspecific effect, consistent with the hypothesis that attention had been drawn to the cued location even when subjects knew it would harm their performance.

\section{EXPERIMENT 4}

The results of Experiments 1-3 reveal performance disruptions for the DIFF condition at only one SOA, $120 \mathrm{msec}$. In Experiment 4, we investigated the time course of the capture effect at SOAs of $40,80,120$, and $200 \mathrm{msec}$ to assess its full magnitude and its development over time. The range of SOAs also provides a more revealing comparison of the CENTER and DIFF conditions. In Experiments 1-3, interference for the CENTER and DIFF conditions was virtually the same. Considering the more foveal location of the flash stimuli in the CENTER condition and the fact that a target never occurred there, it is reasonable to assume that recovery from capture would occur more quickly in the CENTER condition than in the DIFF condition. If so, response times in the two conditions should diverge at some SOA. Finally, if there is both a position-specific effect and a general interference effect produced by cue, it will be possible to examine their time courses separately.

\section{Method}

Targets were presented at four different SOAs measured from the onset of the pretarget flash: $40,80,120$, and $200 \mathrm{msec}$. SOA was varied randomly within blocks. The ALL condition was eliminated. The subjects were tested in one session of 192 trials in each of the remaining four conditions (a total of 768 trials presented in 16 blocks of 48 trials). The displays, order of presentation, and sequence of events were otherwise identical to those in Experiment 3. Sixteen paid volunteers served as subjects.

\section{Results and Discussion}

Figure 4 shows RTs for each condition as a function of SOA. An ANOVA of the mean correct RTs yielded significant main effects of flash condition $[F(3,45)=$ $23.88, p<.001]$ and SOA $[F(3,45)=64.08, p<.001]$ and a significant condition $\times$ SOA interaction $[F(9,135)$ $=8.07, p<.001]$. The error percentages were as follows: NONE, 3.92; SAME, 3.94; CENTER, 4.59; DIFF, 3.99. An ANOVA of the arcsin transform of the square root of the error proportions showed no main effect of condition $[F(3,45)=0.65, p=.59]$, SOA $[F(3,45)=1.26$,

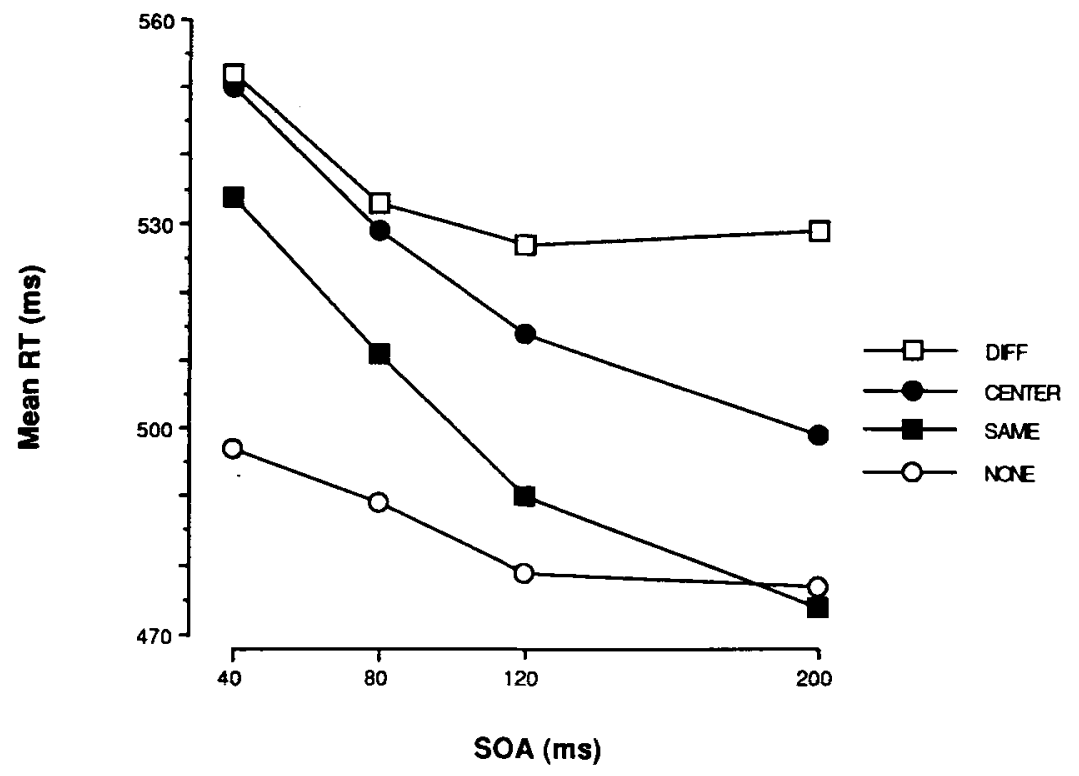

Figure 4. Reaction time as a function of SOA for the four flash conditions of Experiment 4. 
$p=.30]$ and no significant condition $\times \mathrm{SOA}$ interaction $[F(9,135)=1.06, p=.40]$.

The simple effects for RT at each SOA were examined using a Newman-Keuls post hoc test, with $\alpha=.05$. At the 40-msec SOA, latency in the NONE condition was faster than at all other conditions, whereas latencies for the CENTER and DIFF conditions were slower than for the SAME condition. By an SOA of $80 \mathrm{msec}$, RTs for the three flash conditions had decreased approximately $20 \mathrm{msec}$, whereas reaction time for the NONE condition had decreased by only $8 \mathrm{msec}$. At the 120 -msec SOA, all conditions differed reliably. This significant difference was maintained at the 200-msec SOA, except that the SAME and NONE conditions did not differ there.

Substantial interference is found for the DIFF condition at all SOAs. The difference in RT between the NONE and DIFF conditions as a function of increasing SOA (40, 80,120 , and $200 \mathrm{msec}$ ) was $55,44,48$, and $52 \mathrm{msec}$, respectively. The difference in RT between the SAME and DIFF conditions with increasing SOA was 18, 22, 37 , and $55 \mathrm{msec}$, and that for the CENTER and DIFF conditions was $2,4,13$, and $30 \mathrm{msec}$.

Again, the data reveal that involuntary capture of attention (indexed by a substantial RT penalty) cannot be avoided even with substantial incentives and opportunity to ignore an abruptly onset distractor. Of particular interest in Figure 4 is the divergence of the CENTER and DIFF conditions at the 120- and 200-msec SOAs. In Experiments 1-3, the disruption produced by the DIFF condition was only slightly larger than that for the CENTER condition. Their divergence here provides further evidence for spatially specific effects following the presentation of abrupt-onset precues; apparently, it takes longer to redirect attention to the target after it has been drawn away from fixation than after it has been held at fixation (or perhaps "zoomed in"; see Eriksen \& St. James, 1986) by the CENTER flash stimulus. The divergence may also reflect more rapid recovery because of the retinal location of the CENTER flash or the fact that no targets were ever presented there.

The time course of events in Experiment 4 supports our earlier suggestion that both spatially specific and general interference effects influence RTs following the distractor flashes. The general interference can be seen in the improvement of all cued conditions with increasing SOA. ${ }^{4}$ Again, the nature of the effect is unclear and it could be the result of cognitive masking (Eriksen \& Schultz, 1978), filtering cost (Kahneman et al., 1983), or general alerting effects (e.g., Bertelson, 1967) that have been observed in other spatial cuing studies (e.g., Murphy \& Eriksen, 1987; Remington \& Pierce, 1984; Shulman et al., 1979; Tsal, 1983). The spatially specific effects, which are the main focus of this article, are apparent at all SOAs. DIFF RTs show interference relative to SAME RTs at all SOAs and relative to CENTER RTs after $120 \mathrm{msec}$.

Certain aspects of the data from Experiment 4 differ somewhat from those of Experiments 1-3. SAME RTs were significantly slower than NONE RTs at all SOAs except $200 \mathrm{msec}$. Even at the $120-\mathrm{msec}$ SOA, which duplicates the flash-target interval used in Experiments 1-3, NONE RTs were significantly faster than SAME RTs. The difference between the DIFF and CENTER conditions is also somewhat greater here than in Experiments 1-3. These trends are consistent with the hypothesis that the greater temporal uncertainty produced by mixing different flash-target SOA intervals made it both more difficult to ignore the flash stimulus when it was a distraction and more difficult to use it effectively when it correctly predicted target location. To ensure that the results of Experiment 4 reflect the true time course of events, we performed an additional experiment to examine performance at a fixed SOA of $160 \mathrm{msec}$. This provides a direct comparison with Experiments 1-3, which included a fixed but shorter SOA (120 msec).

\section{EXPERIMENT 5}

\section{Method}

Experiment 5 differed from Experiment 4 only in the use of a fixed 160-msec SOA. Eighteen paid subjects drawn from the NASAAmes subject pool served as subjects. None had participated in the earlier experiments.

\section{Results and Discussion}

Table 3 shows the mean RTs and error percentages for each condition. An ANOVA of the correct RTs showed a significant main effect for condition $[F(3,51)=27.29$, $p<.001$ ]. A Newman-Keuls post hoc analysis showed that RTs in the DIFF condition were significantly slower than in all other conditions, including the CENTER condition. In addition, RT in the CENTER condition was significantly slower than that in the SAME or NONE condition. SAME and NONE RTs did not differ significantly. An ANOVA on the arcsin transform of the square root of the error percentages revealed a significant effect of condition $[F(3,51)=3.356, p<.05]$. Newman-Keuls post hoc tests showed that errors in the NONE condition were significantly lower than those in the SAME and CENTER conditions. No other comparisons were significant.

Experiment 5 replicated the key finding of Experiment 4 that latencies in the DIFF condition are slower than in the CENTER condition at long SOAs. This provides converging evidence that the involuntary disruption is actually an involuntary shift of spatial attention toward the flash location, not merely some general disruption. Evidence for position-specific distraction comes from differences in performance between the CENTRAL and DIFF conditions that emerge sometime between $80-160 \mathrm{msec}$ following the

Table 3

Mean Response Time (RT) and Percent Errors (PE) for Each Condition in Experiment 5

\begin{tabular}{|c|c|c|c|c|c|c|c|}
\hline \multicolumn{2}{|c|}{ SAME } & \multicolumn{2}{|c|}{ NONE } & \multicolumn{2}{|c|}{ CENTER } & \multicolumn{2}{|c|}{ DIFF } \\
\hline$\overline{\mathrm{RT}}$ & $\overline{\mathrm{PE}}$ & $\overline{\text { RT }}$ & $\overline{\mathrm{PE}}$ & $\overline{\mathrm{RT}}$ & $\overline{\mathrm{PE}}$ & $\overline{\mathrm{RT}}$ & $\mathrm{PE}$ \\
\hline $489^{a}$ & 5.21 & $495^{\mathrm{a}}$ & 3.78 & $518^{b}$ & 5.52 & $533^{c}$ & 4.58 \\
\hline
\end{tabular}

Note-Response times with the same superscript did not differ significantly from one another. 
flash stimulus. The rate of recovery from the disrupting effects of the flash seems to depend on either the relative locations of the flash and target or the absolute spatial location of the flash. The close correspondence between the results of Experiment 5 and previous experiments confirms that the time course measurements of Experiment 4 reflect the course of development of facilitation and inhibition effects in all the experiments.

\section{GENERAL DISCUSSION}

Using a paradigm designed to maximize the incentive and opportunity for subjects to ignore distracting onset events, we found increases in choice RT when target stimuli were preceded by brief abrupt-onset events at nontarget spatial locations. The interference occurred even though the subjects knew with certainty that the abrupt-onset distractors would always occur at nontarget locations and that failure to ignore them would impair performance. In our experiments, a discrimination response was required to target stimuli that differed from distractor stimuli in elementary feature composition. The distraction condition (DIFF) never required a response to the distractor itself or to a target at the distractor location. Hence, it is not plausible that performance was impaired merely because the subjects had to attend to the distractor in order to reject it as a target (an argument that arises, for example, in the simple reaction time experiments of Posner \& Cohen, 1984). Thus, we conclude that an attentional response to an irrelevant abrupt-onset stimulus is involuntary under the conditions tested here.

The data also revealed that the abrupt-onset flash produced general, spatially nonspecific interference in addition to attentional capture. This nonspecific interference apparently elevated RTs for all flash conditions, especially at short flash-target SOAs. Figure 4 shows RT elevations for all flash conditions at the $40-\mathrm{msec}$ SOA followed by the gradual dissipation of interference, evidenced by RT reductions for all cued conditions over the first 80 $120 \mathrm{msec}$ following flash onset. These reductions are found not only in the absolute latencies for each condition, but relative to the NONE condition as well, which has no flash. A comparison of Experiment 1 with Experiment 2 further reveals that RTs for all flash conditions are elevated when the onset time of the flash is less predictable, consistent with a spatially nonspecific source of interference.

One of the most striking consequences of this nonspecific interference is the failure to find substantial benefits of the SAME condition relative to the NONE condition. The subjects were apparently using the flash in the SAME condition to focus attention on the target location. SAME RTs were consistently faster than either CENTER or DIFF times, and, at long SOAs, they were somewhat faster than NONE times. We assume that the benefit derived from the spatial information in the flash was masked by the nonspecific interference from the flash, which slowed responses overall. Similar effects have been observed in a variety of experiments. For example, Erik- sen and Schultz (1978) cite a number of studies in which RT to a target character is elevated when flanked by unrelated characters, colored forms, or replicas of the target itself. They call this effect cognitive masking and attribute it to capacity limitations in visual information processing. Similar results have been called filtering costs by Kahneman et al. (1983), who argue that they result from disruptions in the deployment of attention caused by the presence of extraneous characters.

\section{Models of Attentional Capture}

We briefly consider a model that, unlike cognitive masking or filtering cost accounts, relates the pattern of results in our experiments directly to the process of attentional capture. According to the model, the onset of the flash directs attention not to the target location per se, but to a larger region delineated by the contours of the flash stimulus surrounding one of the boxes (initial shift). The onset of the target then redirects attention precisely to the target character (corrective shift). However, this second shift can only be made after attention is first disengaged from its initial deployment. Thus, target processing will be delayed when the target appears before the completion of the initial attentional shift to the flash stimulus. With increasing flash-target SOA, there is an increasing likelihood that the initial attentional shift has been completed before target onset, accounting for the improvements in RT across the early SOAs for the CENTER, SAME, and DIFF conditions in Experiment 4 (see Figure 4).

Because even in the SAME condition the initial shift is to the flash stimulus and not to the adjacent potential target location, the initial shift does not optimally position attention for target processing. On some SAME trials, this might be sufficient to permit target identification, but, on some proportion of the trials, a refinement of the attentional set, such as further focusing (see Eriksen \& St. James, 1986) is required upon target onset. In the NONE condition, however, the target itself is the initial attentional focus, and no further adjustment is ever necessary. Thus, the model can explain the absence of benefit (or small cost) for SAME responses with respect to NONE RTs at short SOAs.

There are various ways in which the advantage of the SAME condition over the CENTER and DIFF conditions can be accounted for by the model. For example, the assumption that, on some proportion of SAME trials, the target can be identified without a corrective attentional shift would produce faster SAME RTs than those in the CENTER and DIFF conditions, where corrective shifts would always be required. It is also possible that small corrections in the locus of spatial attention can be made more efficiently than the relatively large corrections needed in the CENTER and DIFF conditions, perhaps without the necessity for a separate disengage-engage sequence. Thus, the DIFF condition would require that attention first be engaged at a nontarget location (flash location), then be disengaged from this location, and, finally, be shifted to and engaged at the target location, whereas the SAME condition would require only the ini- 
tial engage and perhaps a corrective shift or refining focus. This model makes two assumptions that have interesting implications for attention control mechanisms: (1) a shift of spatial attention, once begun, cannot be interrupted, and (2) a shift outside the focus of attention is more difficult than an adjustment of that focus. ${ }^{5}$ Further experimentation will be required to establish whether or not these assumptions are valid.

We have demonstrated that attention will be drawn involuntarily to an abrupt-onset stimulus even when it is a completely irrelevant distractor that subjects are motivated to ignore. In our experiments, the flash stimuli occurred while the subjects were awaiting a target in an unknown position. Under these circumstances, subjects evidently cannot exert the direct control over the allocation of spatial attention necessary to prevent attending to the abrupt onset. In contrast, Yantis and Jonides (1990) have shown that an attentional response to a completely irrelevant abrupt-onset distractor is not mandatory when attention is already focused on a location that is both marked by a previously displayed stimulus and guaranteed to later contain a target. Spatial set alone is not sufficient to avoid capture; the target location must be known with certainty (Müller \& Rabbitt, 1989; Yantis \& Jonides, 1990). This pattern of results suggests that when the spatial filtering of unwanted information can be accomplished, the control is not carried out by a direct voluntary negative set (do not attend to any $X$ ), but by establishing a positive focal attentional set that serves to insulate attention from the effects of new stimuli occurring at other locations. Some property of focused spatial attention is apparently responsible for the absence of an attentional response to events outside the attentional focus, including those that would otherwise involuntarily capture attention.

One possible reason why stimuli outside the narrow focus of attention do not involuntarily summon attention is that they are filtered out by an exceptionally fast earlyselection blockage (Yantis \& Johnston, 1990). When attention can be focused completely on a specified region of the visual field, as in Yantis and Jonides (1990), the filtering of information outside that region might be great enough that a stimulus that would normally elicit an involuntary attentional response never impinges on the attentional mechanism with sufficient strength. According to this early-filter explanation, abrupt onsets in the present experiments could not be ignored because the absence of a focused attentional set allowed the effective stimulus (the abrupt onset) to activate an attentional response.

An alternative account is that the stimulus events may be processed, but when the spatial attention mechanism is in a highly focused state, it is locked in a mode that prevents it from responding to events outside that focus. According to the locked-mode explanation, our results arise because the need to keep spatial attention diffuse and ready to move to the as-yet-unknown target location is incompatible with putting it in a locked mode to prevent it from responding to the flash stimulus. Further experiments can distinguish between the stimulus-blocking and response-locking hypotheses.
Our results are clearly consistent with the automatic capture of attention by abrupt onsets. Yantis and Jonides (1990) argue that attentional capture satisfies other criteria automaticity as well. In our experiments, though, both flash and target have an abrupt onset. If the abrupt onset of the target was used to locate and shift attention to it, then subjects were being asked to use the second of two closely timed onsets, ignoring the first. It may not be possible to change attentional control settings so rapidly. Rather, attention may been captured by the abrupt onset of the flash because it had been set to respond to the abrupt onset of the target. If so, it raises the possibility that automaticity, and involuntariness, are not solely determined by external stimulus properties but are sensitive to internal control settings. Like a programmable interrupt in a computer, external stimuli with properties matching those for which attention is set would be attended to, and the same properties would be ignored with other control settings. Folk, Remington, and Johnston (in press) examine these possibilities further.

\section{Conclusion}

We have shown here a fundamental limitation on the conscious control of spatial attention. Our experiments reveal conditions under which the control of spatial attention is completely involuntary: attention is captured by an irrelevant event despite subjects' intentions to ignore the event. Unlike previous investigations, our paradigm provided strong incentives to ignore the distracting abrupt onset, but these were insufficient to prevent capture. Together with the results of Yantis and Jonides (1990) and Müller and Rabbitt (1989), our findings lead us to speculate that voluntary control of attention is limited to focusing attention in advance on locations, objects, or properties of interest. Direct inhibitory control of spatial attention-commanding it not to respond-is not effective under the conditions studied here, and may not be possible.

\section{REFERENCES}

BASHINSKI, H. S., \& BACHARACH, V. R. (1980). Enhancement of perceptual sensitivity as the result of selectively attending to spatial locations. Perception \& Psychophysics, 28, 241-280.

BERTEISON, P. (1967). The time course of preparation. Quarterly Joumal of Experimental Psychology, 19, 272-279.

Bushnell, M. C., Robinson, D. L., \& Goldberg, M. E. (1978). Dissociation of movement and attention: Neuronal correlates in parietal cortex. Neurosciences Abstracts, 4, 621.

Duncan, J. (1984). Selective attention and the organization of visual information. Journal of Experimental Psychology: General, 113, 501-517.

ERIKSEN, C. W., \& Hoffman, J. E. (1972). Temporal and spatial characteristics of selective encoding from visual displays. Perception \& Psychophysics, 12, 201-204.

ERIKSEN, C. W., SChultz, D. W. (1978). Temporal factors in visual information processing: A tutorial review. In J. Requin (Ed.), Attention and performance VII (pp. 3-24). Hillsdale, NJ: Erlbaum.

ERIKSEN, C. W., ST. JAMES, J. D. (1986). Visual attention within and around the field of focal attention: A zoom lens model. Perception \& Psychophysics, 40, 225-240.

ERIKSEN, C. W., \& YEH, Y. (1985). Allocation of attention in the visual field. Joumal of Experimental Psychology: Human Perception \& Performance, 11, 583-597. 
Folk, C. L., Remington, R. W., Johnston, J. C. (in press). Involuntary covert orienting is contingent on attention control settings. Joumal of Experimental Psychology: Human Perception \& Performance.

Hawkins, H. L., Hielyard, S. A., Luck, S. J., Mouloua, M., Downing, C. J., Woodward, D. P. (1990). Visual attention modulates signal detectability. Journal of Experimental Psychology: $\mathrm{Hu}$ man Perception \& Performance, 16, 802-811.

Hoffman, J. E., Nelson, B. (1981). Spatial selectivity in visual search. Perception \& Psychophysics, 30, 283-290.

JoNIDES, J. (1981). Voluntary versus automatic control over the mind's eye's movement. In J. B. Long \& A. D. Baddeley (Eds.), Attention and performance IX (pp. 187-203). Hillsdale, NJ: Erlbaum.

JoNIDES, J., MACK, R. (1984). On the cost and benefit of cost and benefit. Psychological Bulletin, 96, 29-44.

Jonides, J. Naveh-Benjamin, M., \& Palmer, J. (1985). Assessing automaticity. Acta Psychologica, 60, 157-171.

Kahneman, D., Treisman, A. (1984). Changing views of attention and automaticity. In R. Parasuraman, R. Davies, \& J. Beatty (Eds.), Varieties of attention (pp. 29-61). New York: Academic Press.

Kahneman, D., Treisman, A., \& Burkell, J. (1983). The cost of visual filtering. Joumal of Experimental Psychology: Human Perception \& Performance, 9, 510-522.

LABERGE, D. (1981). Automatic information processing: A review. In J. B. Long \& A. D. Baddeley (Eds.), Attention and performance IX (pp. 173-186). Hillsdale, NJ: Erlbaum.

Lambert, A., Spencer, E., Mohindra, N. (1987). Automaticity and the capture of attention by a peripheral display change. Current Psychological Research \& Reviews, 6, 136-147.

MilLER, J. (1989). The control of attention by abrupt visual onsets and offsets. Perception \& Psychophysics, 45, 567-571.

MüLLER, H. J., \& FINDLAY, J. M. (1987). Sensitivity and criterion effects in the spatial cuing of visual attention. Perception \& Psychophysics, 42, 383-399.

MülleR, H. J., RABBITT, P. M. A. (1989). Reflexive and voluntary orienting of visual attention: Time course of activation and resistance to interruption. Joumal of Experimental Psychology: Human Perception \& Performance, 15, 315-330.

MuRPhy, T. D., \& ERIKSEN, C. W. (1987). Temporal changes in the distribution of attention in the visual field in response to precues. Perception \& Psychophysics, 42, 576-586.

Posner, M. I. (1978). Chronometric explorations of mind. Hillsdale, NJ: Erlbaum.

POSNER, M. I. (1980). Orienting of attention. Quarterly Journal of Experimental Psychology, 32, 3-25.

Posner, M. I., \& CoHEN, Y. (1984). Components of visual orienting. In H. Bouma \& D. G. Bouwhuis (Eds.), Attention and performance $X$ (pp. 55-66). Hillsdale, NJ: Erlbaum.

Posner, M. I., SNyder, C. R. R., \& Davidson, B. J. (1980). Attention and the detection of signals. Journal of Experimental Psychology: General, 109, 160-174.

REMINGTON, R. W. (1980). Attention and saccadic eye movements. Journal of Experimental Psychology: Human Perception \& Performance, 6, 726-744.

Remington, R. W., \& Pierce, L. (1984). Moving attention: Evidence for time-invariant shifts of visual selective attention. Perception \& Psychophysics, 35, 393-399.

SHIFFrIN, R. M., \& SCHNEIDER, W. (1977). Controlled and automatic human information processing: II. Perceptual learning, automatic attending, and a general theory. Psychological Review, 84, 127-190.

Shulman, G. L., Remington, R. W., \& Mclean, J. P. (1979). Moving attention through visual space. Joumal of Experimental Psychology: Human Perception \& Performance, 5, 522-526.
Treisman, A., Gormican, S. (1988). Feature analysis in early vision: Evidence from search asymmetries. Psychological Review, 95 , 15-48.

TSAL, Y. (1983). Movements of attention across the visual field. Journal of Experimental Psychology: Human Perception \& Performance, 9, 523-530.

WARNer, C. B., Juola, J. F., \& Koshino, H. (1990). Voluntary allocation versus automatic capture of visual attention. Perception \& Psychophysics, 48, 243-251.

YANTIS, S., \&ohNSTON, J. C. (1990). On the locus of visual selection: Evidence from focused attention tasks. Journal of Experimental Psychology: Human Perception \& Performance, 16, 135-149.

YANTIS, S., \& JonIDES, J. (1984). Abrupt visual onsets and selective attention: Evidence from visual search. Joumal of Experimental Psychology: Human Perception \& Performance, 10, 601-621.

YANTIS, S., \& Jonides, J. (1990). Abrupt visual onsets and selective attention: Voluntary versus automatic allocation. Joumal of Experimental Psychology: Human Perception \& Performance, 16, 121-134.

\section{NOTES}

1. Duncan (1984) has argued that attention is focused on objects rather than spatial locations. The question of whether visual selective attention is spatially based or object based, though important, is not critical to the issue of its control as addressed by this paper. Our results can be recast into an object-based framework by assuming that attention is drawn to the abrupt-onset stimulus, or some other nearby object, without substantially altering the conclusions.

2. Miller (1989) has shown that the abrupt offset of a stimulus has a similar, if somewhat weaker, ability to involuntarily summon attention, suggesting that the transient nature of the stimulus is the effective feature. For simplicity, we will refer to the attention-summoning properties of abrupt onsets rather than to visual transients.

3. A number of analyses were carried out to ensure that the distraction effect was not the result of presentation order or an artifact resulting from the combination of target location, cue location, and response hand. In no study was presentation order significant. In Experiment 1, there was no effect of response-hand assignments. In all experiments, standard stimulus-response compatibility effects were obtained. However, the same basic pattern of RTs across conditions was observed at each target location, the effects differing somewhat in magnitude. The distraction effect is robust. It is not an artifact of stimulus-response compatibility or some unusual feature of one of the display locations.

4. There is a small $(15 \mathrm{msec})$ decrease in RT for the NONE condition over the first $120 \mathrm{msec}$. This decrease probably reflects the waning influence of the alerting interval that began with the blinking of the fixation cross.

5. This account has been framed in terms of space-based model of spatial attention. A similar argument could be made within an objectbased approach to spatial attention (e.g., Duncan, 1984). It might be assumed, for example, that since the flash stimulus was so brief, the initial shift left attention focused on the box. The advantage for the SAME condition over the CENTER and DIFF conditions could have resulted from the target's appearing on an attended rather than an unattended object. A separate attentional shift to the target would still be required as described in the text.

(Manuscript received September 14, 1990; revision accepted for publication November 4,1991 .) 